\title{
Is NerBloc, the botulinum toxin type B formulation, an effective therapy for cervical dystonia?
}

\author{
Masahiro Horiuchi ${ }^{1 *}$ Akira Endo ${ }^{2}$ Mika Ishii $^{2}$ and Ryuji Kaji ${ }^{3}$ \\ ${ }^{1}$ Department of Neurology, Kawasaki Municipal Tama Hospital, Kawasaki, Japan \\ ${ }^{2}$ Eisai Corporation, Tokyo, Japan \\ ${ }^{3}$ Department of Clinical Neuroscience, Institute of Health Biosciences, Tokushima University Graduate School, Tokushima, Japan
}

\begin{abstract}
Background: To test the efficacy of NerBloc, a botulinum toxin type B (BTB) for patients suffering from cervical dystonia.

Methods: Patients (77) with cervical dystonia that was difficult to treat with only oral medications were enrolled in this study. Drug efficacy and the duration of efficacy were assessed by measuring the overall improvements in disease severity, disability, and pain associated with each administration of BTB. We also assessed the effect of BTB administration in ten patients who switched to BTB from botulinum toxin type A (BTA), as evaluated by the Toronto Western Spasmodic Torticollis Rating Scale (TWSTRS).

Results: Four episodes of adverse effects were observed. Overall improvements were assessed by measuring disease severity, disability, and pain. There were no patients whose condition worsened or remained unchanged. The average duration of efficacy per administration was independent of the patient's history of treatment with BTA.
\end{abstract}

However, the severity of symptoms, disability, and pain, as assessed by TWSTRS scores, improved in patients who switched from BTA usage to BTB.

Discussion: BTB is an effective therapy for cervical dystonia.

\section{Introduction}

We found that many individuals suffered a financial burden and psychological stress because of dystonia. Improving the quality of life of patients with cervical dystonia by reducing these burdens is a key aspect in the clinical management of this condition [1].

In 2013, NerBloc, a botulinum toxin type B (BTB) neuromuscularblocking agent, was launched in Japan, resulting in a significant addition to the options available for the medical treatment of cervical dystonia. Additionally, the low cost of NerBloc is thought to be a major benefit for the patients with cervical dystonia. This study aim to evaluate the efficacy and the safety of BTB and the efficacy of switching from botulinum toxin type A (BTA) to BTB.

BTB and BTA are equally efficacious in the treatment of cervical dystonia, as shown by a randomized double-blind comparison study (non-inferiority trial) [2]. Based on the Toronto Western Spasmodic Torticollis Rating Scale (TWSTRS) scores, BTA and BTB formulations achieved similar durations of efficacy (13.1 and 13.7 weeks, respectively). Analysis of the TWSTRS [3] scores in a Japanese, comparative, double-blind, placebo-controlled study of BTB (4 weeks after dosing) confirmed the efficacy of BTB in terms of reduction of severity, disability, and pain [4]. Therefore, BTB could become a good choice for the treatment of cervical dystonia.

\section{Materials and methods}

This study was post marketing product surveillance (open-label) by Eisai Corporation (NB01S) and retrospectively evaluated either the efficacy or the safety of a BTB formulation (NerBloc) in patients with cervical dystonia. The study involved 77 adult patients (mean age 44.6 years, 38 men, 39 women) who were administered BTB at Kawasaki Municipal Tama Hospital between July 1, 2014, and June 30, 2015. They were hard to treat by only oral medication. Written informed consent for participation in the study was obtained from participants before start of the study. This study received an ethical approval from our institutional review board (D323 Tama).

The patient characteristics, including previous BTA usage and the BTB discontinuation rate, are shown in Table 1. The efficacy of BTB was assessed by assessing the overall improvement in disease severity, disability, and pain. The duration of efficacy was assessed by the patients' feedback.

Additionally, the efficacy of BTB was evaluated in ten patients who switched to BTB after having been treated with BTA. This study was post marketing product surveillance by Eisai Corporation (NB02T). A gradual switching from BTA to BTB (mainly due to the economic

Correspondence to: Masahiro Horiuchi, Department of Neurology Kawasaki Municipal Tama Hospital, 1-30-37 Shukugawara, Tama-Ward, Kawasaki, Kanagawa 214-8525, Japan, Tel: +81-44-933-8111; Fax: +81-44-933-8362; E-mail:m2horiuchi@marianna-u.ac.jp

Key words: Botulinum toxin type A, Botulinum toxin type B, Cervical dystonia, NerBloc, Toronto Western Spasmodic Torticollis Rating Scale (TWSTRS)

Received: September 29, 2016; Accepted: October 13, 2016; Published: October 17,2016 
Table 1. Patient characteristics including previous BTA usage and BTB discontinuation status BTA: botulinum toxin type A; BTB: botulinum toxin type B

\begin{tabular}{|c|c|c|c|c|}
\hline & & & Patients & Percentage \\
\hline \multicolumn{3}{|c|}{ Patients assessed (n) } & 77 & $100.0 \%$ \\
\hline \multirow[t]{2}{*}{ Gender } & \multicolumn{2}{|l|}{ Male } & 38 & $49.4 \%$ \\
\hline & \multicolumn{2}{|l|}{ Female } & 39 & $50.6 \%$ \\
\hline \multicolumn{3}{|l|}{ Mean age } & \multicolumn{2}{|c|}{44.6 years old } \\
\hline \multirow[t]{2}{*}{ BTA usage } & \multicolumn{2}{|c|}{ Not used } & 39 & $50.6 \%$ \\
\hline & \multicolumn{2}{|l|}{ Used } & 38 & $49.4 \%$ \\
\hline \multirow{7}{*}{$\begin{array}{l}\text { Discontinuation } \\
\text { of BTB }\end{array}$} & \multicolumn{2}{|c|}{ Not discontinued } & 49 & $63.6 \%$ \\
\hline & \multicolumn{2}{|c|}{ Discontinued } & 28 & $36.4 \%$ \\
\hline & \multirow[t]{5}{*}{ Reason } & Failure to attend hospital visits & 12 & $42.9 \%$ \\
\hline & & Adverse events & 1 & $3.6 \%$ \\
\hline & & Lack of efficacy from starting dose & 3 & $10.7 \%$ \\
\hline & & Disappearance of symptoms & 2 & $7.1 \%$ \\
\hline & & Others & 8 & $28.7 \%$ \\
\hline
\end{tabular}

reason) was achieved in these ten patients by assessing the TWSTRS scores after each dose and at 4 weeks after administration. Drug efficacy was assessed for 1 year from the initial administration. Written informed consent for participation in the study was obtained from participants before start of the study. This study received an ethical approval from our institutional review board (D324 Tama).

\section{Results}

Four episodes of adverse effects were observed in 3 of 77 patients (3.9\%). Dysphagia was observed in 2 patients $(3.0 \%)$, and dry mouth and hoarseness were observed in 1 patient (1.5\%). All the adverse reactions were non-serious and ultimately resolved in about one week. All cases improved naturally. Overall improvements were assessed by measuring disease severity, disability, and pain. There were no patients whose condition worsened or remained unchanged Figure 1. The average duration of efficacy per administration was independent of the patient's history of treatment with BTA. The average duration of efficacy per administration was between 6.3 and 8.2 weeks and was independent of the patient's history of treatment with BTA.

However, the severity of symptoms, disability, and pain, as assessed by TWSTRS scores, improved in the patients who switched from BTA to BTB (Figure 2). A long-term improvement was observed in the mean TWSTRS scores of the 10 patients who switched from BTA to BTB. Of these 10 patients, 8 of the 9 patients in whom pain scale scores were recorded, reported improvement in their pain score, whereas the pain score in the other patient remained unchanged. Eight of the 9 patients also reported improvement in the total TWSTRS score, whereas the score in the other patient remained unchanged. No exacerbation in pain scale or total score was observed in the 10 patients who switched from BTA to BTB (Table 2).

\section{Discussion}

Before this study, to clarify their illness history and problems encountered in daily life, a questionnaire survey was conducted in 232 patients with dystonia [1]. Among the 169 patients who responded, 74 patients suffered from cervical dystonia. All 169 patients reported that they regularly experienced inconvenience in daily life and had concerns about finances and employment. Many of these patients expressed the opinion that the social security system should be improved [1].

Brashear et al. [5] carried out a comparative, double-blind, placebo-controlled study of BTA versus BTB in the cervical dystonia patients who were responsive to BTA treatment. Their study involved

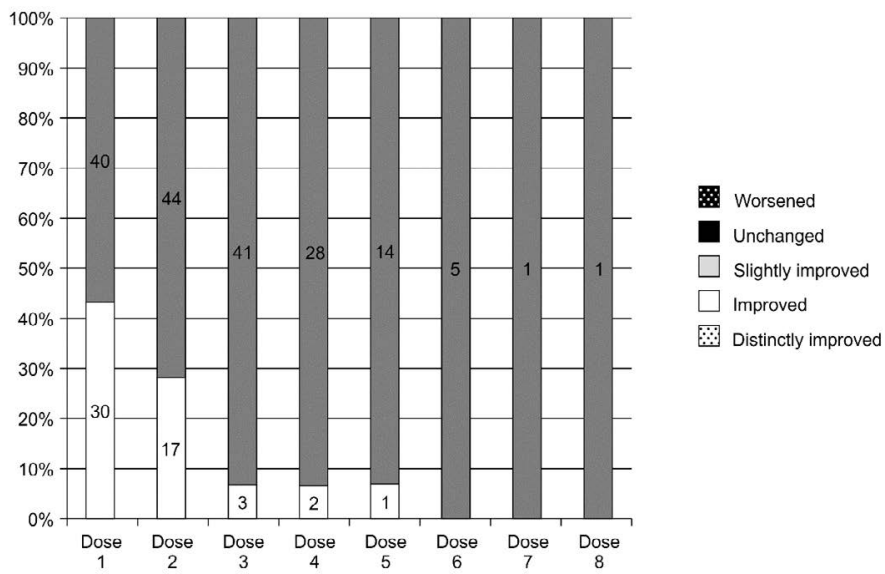

Figure 1. Overall improvements in disease severity, disability, and pain in 77 cervical dystonia patients administered BTB

The numbers above the bars indicate number of patients receiving the particular dose. There were no patients whose condition worsened or remained unchanged. BTB: botulinum toxin type B

Table 2. TWSTRS scores for the ten patients who switched from BTA to BTB TWSTRS Toronto Western Spasmodic Torticollis Rating Scale; BTA: botulinum toxin type A; BTB: botulinum toxin type B.

\begin{tabular}{|c|c|c|c|c|c|c|c|}
\hline & \multirow[t]{2}{*}{ Sex } & \multirow[t]{2}{*}{ Age (y) } & \multirow[t]{2}{*}{ Doses } & \multicolumn{2}{|c|}{ Pain scale } & \multicolumn{2}{|c|}{ Total score } \\
\hline & & & & $\begin{array}{c}\text { Starting } \\
\text { scale }\end{array}$ & $\begin{array}{l}\text { Last } \\
\text { scale }\end{array}$ & $\begin{array}{c}\text { Starting } \\
\text { scale }\end{array}$ & $\begin{array}{l}\text { Last } \\
\text { scale }\end{array}$ \\
\hline 009 & $\mathrm{~F}$ & 45 & 4 & 12 & 9 & 45 & 39 \\
\hline 010 & $\mathrm{~F}$ & 39 & 4 & 10 & 8 & 49 & 42 \\
\hline 012 & $\mathrm{M}$ & 38 & 1 & 8 & - & 42 & - \\
\hline 013 & M & 33 & 3 & 8 & 8 & 39 & 39 \\
\hline 014 & $\mathrm{~F}$ & 29 & 5 & 7 & 7 & 33 & 28 \\
\hline 015 & M & 61 & 6 & 7 & 6 & 34 & 28 \\
\hline 016 & $\mathrm{~F}$ & 49 & 4 & 10 & 8 & 34 & 30 \\
\hline 017 & $\mathrm{~F}$ & 52 & 2 & 8 & 7 & 46 & 42 \\
\hline 031 & $\mathrm{~F}$ & 45 & 4 & 12 & 8 & 45 & 37 \\
\hline 032 & M & 35 & 2 & 6 & 6 & 36 & 33 \\
\hline
\end{tabular}

109 cervical dystonia patients who were administered either 5,000 or 10,000 units of drug as a single dose into multiple tonic muscles; the total TWSTRS scores were evaluated. They observed significant differences in the total TWSTRS scores 4 weeks after administration in both drug groups compared to the placebo groups. They also reported significant differences in the pain assessment scores 4 weeks after 


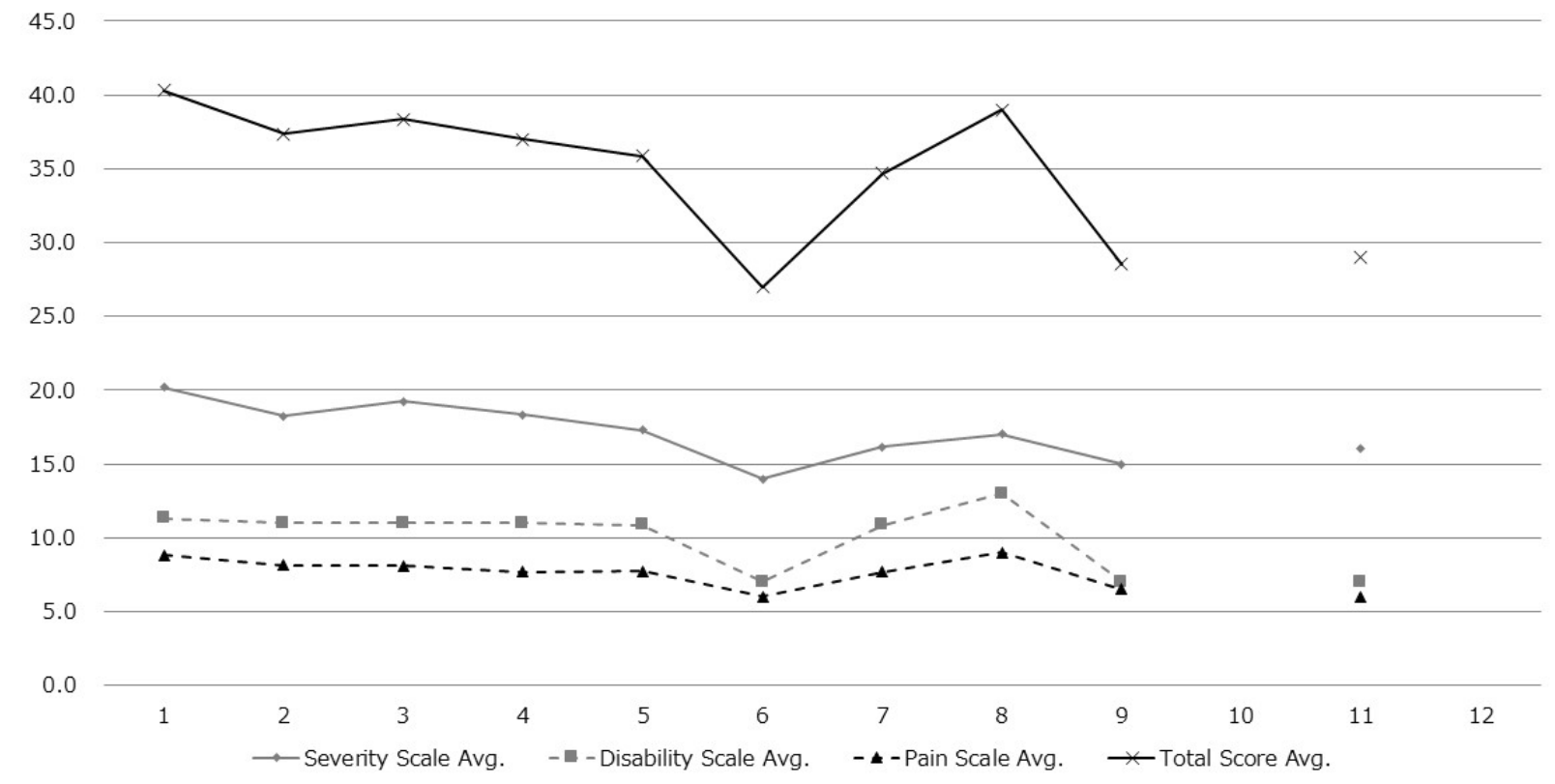

Figure 2. Variation in mean TWSTRS scores of patients that switched from type A botulinum usage Improvement was observed in type A switched patients, by gradually continuing with type B. TWSTRS: Toronto Western Spasmodic Torticollis Rating Scale

administration (the visual analog scale and the TWSTRS pain score) compared to those of the placebo groups.

Brin et al. [6] carried out a double-blind, placebo-controlled study of BTB in cervical dystonia patients resistant to BTA treatment. In their study, 10,000 units of BTB was administered as a single dose into multiple tonic muscles in 77 cervical dystonia patients resistant to BTA treatment; the total TWSTRS scores were evaluated 4 weeks after administration. They observed significant statistical differences in the total TWSTRS scores and in three visual analog pain scores between the drug and placebo groups 4 weeks after dosing.

The evidence for the efficacy of each formulation in the patients resistant to other treatments is clear. Given that BTA is ineffective in $5 \%-10 \%$ of patients, switching to BTB may be effective for such patients [7]. Even in patients who have developed antibodies to BTA, BTB is still efficacious [8].

Therefore, BTB formulations could become a good choice for treatment of cervical dystonia. Assuming that 100 units of Botox, a BTA formulation, is equivalent to 5,000 units of NerBloc, a BTB formation $[9,10]$, the use of BTB would greatly reduce the cost (possibly by as much as $40 \%$ compared to that incurred by BTA). Both BTA and BTB treat cervical dystonia effectively (non-inferiority was confirmed). ${ }^{4}$ Furthermore, BTB relieves pain more effectively than BTA does [11].

\section{Conclusions}

Four episodes of adverse effects were observed. A same proportion of patients with no history of BTA usage reported improvement and did those with a history of BTA usage. However, the severity of symptoms, disability, and pain, as assessed by TWSTRS scores, improved in patients who switched from BTA usage to BTB.

NerBloc, a BTB formulation, is as effective as BTA for treating cervical dystonia, and it has the potential to significantly reduce the financial burden for patients, which is one of the most serious issues affecting dystonia patients. Consequently, NerBloc could become a good choice for the treatment of cervical dystonia.

\section{Authorship statement}

Research Project: AE, MI, and RK designed the study

Statistical Analysis: MH collected and analyzed the data.

Manuscript: MH drafted the manuscript; All authors reviewed and approved the final manuscript.

\section{Acknowledgements}

None.

\section{References}

1. Horiuchi M, Kawashima S, Mochizuki K, Satoh T (2016) Economic and employment issues in patients with dystonia: a self-report questionnaire survey. $J$ Neurol Neurosci 7: 95 .

2. Pappert EJ, Germanson T (2008) Myobloc/Neurobloc European Cervical Dystonia Study Group. Botulinum toxin type B vs. type A in toxin-naïve patients with cervical dystonia: randomized, double-blind, noninferiority trial. Mov Disord 23: 510-517.

3. Consky ES, Lang AE (1994) Assessment of cervical dystonia: The Toronto Western Spasmodic Torticollis Rating Scale (TWSTRS). In: Jankovic J, Hallet M (Eds.,). Therapy with botulinum toxin, neurological disease and therapy series. New York: Marcel Dekker. pp. 224-226.

4. Kaji R, Shimizu H, Takase T, Osawa M, Yanagisawa N (2013) A double-blind comparative study to evaluate the efficacy and safety of NerBloc (rimabotulinumtoxinB) administered in a single dose to patients with cervical dystonia. Brain Nerve 65: 203-211.

5. Brashear A, Lew MF, Dykstra DD, Comella CL, Factor SA, et al. (1999) Safety and efficacy of NeuroBloc (botulinum toxin type B) in type A-responsive cervical dystonia. Neurology 53: 1439-1446. [Crossref]

6. Brin MF, Lew MF, Adler CH, Comella CL, Factor SA, et al. (1999) Safety and efficacy of NeuroBloc (botulinum toxin type B) in type A-resistant cervical dystonia. Neurology 53: 1431-1438. [Crossref]

7. Costa J, Borges A, Espirito-Santo C, Castelão M, Ferreira J, et al. (2005) Botulinum 
toxin type A versus botulinum toxin type B for cervical dystonia. Cochrane Database Syst Rev 1: CD004314.

8. Dressler D, Bigalke H, Benecke R (2003) Botulinum toxin type B in antibody-induced botulinum toxin type A therapy failure. J Neurol 250: 967-969. [Crossref]

9. Comella CL, Jankovic J, Shannon KM, Tsui J, Swenson M, et al. (2005) Comparison of botulinum toxin serotypes A and B for the treatment of cervical dystonia. Neurology 65: 1423-1429. [Crossref]
10. Tintner R, Gross R, Winzer UF, Smalky KA, Jankovic J (2005) Autonomic function after botulinum toxin type A or B: a double-blind, randomized trial. Neurology 65 765-767. [Crossref]

11. Lew MF, Chinnapongse R, Zhang Y, Corliss M (2010) RimabotulinumtoxinB effects on pain associated with cervical dystonia: results of placebo and comparator-controlled studies. Int J Neurosci 120: 298-300. [Crossref]

Copyright: (C2016 Horiuchi M. This is an open-access article distributed under the terms of the Creative Commons Attribution License, which permits unrestricted use, distribution, and reproduction in any medium, provided the original author and source are credited. 